\title{
Enhancing the Optical Properties of Polyvinyl Alcohol by Blending It with Polyethylene Glycol
}

\author{
R.M. Ahmed*, A.A. Ibrahim And E.A. El-Said \\ Physics Department, Faculty of Science, Zagazig University 44519, Zagazig, Egypt \\ (Received July 13, 2019; revised version October 25, 2019; in final form November 27, 2019)
}

\begin{abstract}
In the present research work a solution casting technique was utilized to fabricate various blends of polyvinyl alcohol (PVA)/polyethylene glycol (PEG). Embedding PEG in PVA resulted in obvious shifts in the absorption edge which points to a decrease in the energy gap values based on PEG content. Loss of the photon energy was a consequence of increasing the extinction coefficient at high wavelengths. The PEG content increased the refractive index of the PVA in the produced PVA/PEG blends. The direct and indirect energy gap values were decreased whereas the Urbach energy values were increased with increasing the PEG content. The exact electron transition which is responsible for the absorption process was determined successfully based on the complex optical dielectric functions. In addition, some other optical parameters were estimated to more understand fully the effect of embedding the PEG in the PVA. The Fourier transform infra red spectroscopy was used to determine the characteristic functional groups of both the PVA and the PEG.
\end{abstract}

DOI: 10.12693/APhysPolA.137.317

PACS/topics: optical conductivity, optical band gap, Urbach energy, polyvinyl alcohol/polyethylene glycol, blends

\section{Introduction}

Recently, the improvement of polymeric materials by modification of their physical properties has become of concern in order to fulfil the increasing requirements in various industrial applications such as cables, materials of electronic packaging, and jackets, and films of capacitors [1]. Light weight, reprocessability and low density are common features of the polymer matrices of concern. In addition, the optical properties of polymers, copolymers, polymer blends and polymer composites have received considerable attention resulting in increased understanding of their structures and the possible applications in various optical devices and industrial applications [1,2].

In the past decade polymer blends have become increasingly used commercially and industrially, surpassing copolymers and also homopolymers [3]. Physically, mixing two polymers or more is known as the process of blending which results in modification of the structure of the homopolymers used with lower cost compared to synthesising of a new material. For instance, easy preparation and developing the electrical conductivity were a few of the advantages of a polymer blending process of PVA and PEG [4].

Due to its interesting optical properties, PVA is considered as one of the most significant commercial polymers. The instability of the vinyl alcohol monomer with respect to acetaldehyde is the reason PVA is prepared by first polymerizing vinyl acetate followed by converting the produced polyvinyl acetate to PVA [5]. Because of its high refractive index, excellent elasticity, lack of color and low cost, PVA has been utilized as a nonlinear optical element, a polymer waveguide and for the replacement of glass in its uses in industrial applications. In addition, the development of PVA based complexes can be achieved effectively due to the existence of hydroxyl groups on the carbon chain backbone of PVA which provides a source for hydrogen bonding that facilitates this process. In order to enhance the application of PVA in the pharmaceutical and clinical fields, polyethylene glycol (PEG) is a promising candidate to blend with PVA to improve its physical properties [6].

PEG has a number of useful features, having good protein adsorption resistance, superb water solubility, low immunogenicity and low toxicity. PEG causes no damage when it contacts with the lips or skin; therefore it is suitable to be used in various applications as a coating material for biotechnical applications [7]. Also, it can be used as a binder in the preparation of ceramics and its significant physical and chemical properties enable it to be used in various applications in industry, e.g., $[4,8]$.

In this study a solution casting method was used to prepare PVA/PEG blends with various contents of the components. The casting technique was chosen because it is an easy and a simple method, cheap, producing a uniform thickness distribution and providing the maximum optical purity. The produced samples were characterized by Fourier transform infra red spectroscopy (FTIR). The reflection and absorption spectra were utilized to estimate some required optical parameters to understand the effect of embedding PEG in PVA on their structure and optical properties. 


\section{Material and methods}

\subsection{Sample preparation}

$\mathrm{PVA}\left(M_{w} \approx 115,000 \mathrm{~g} / \mathrm{mol}\right)$ was purchased from LOBA CHEMIE PVT. Ltd., India, while PEG $\left(M_{w} \approx 4000 \mathrm{~g} / \mathrm{mol}\right)$ was obtained from Fluka Chemical Co., Germany. These two homopolymers were used without further purifications. A solution casting method was used to prepare the films of the samples. Powder of the PVA was dissolved in distilled water at $80^{\circ} \mathrm{C}$ with gentle stirring for $120 \mathrm{~min}$ then left to cool to room temperature. The PEG powder was dissolved in distilled water at room temperature for $60 \mathrm{~min}$. The solutions of $\mathrm{PEG}_{100-x}$ were added slowly to the $\mathrm{PVA}_{x}$ solution with different ratios under stirring for $60 \mathrm{~min}$. Consequently, the solutions of the blends were poured into glass dishes and placed in a vacuum at $45^{\circ} \mathrm{C}$ for $48 \mathrm{~h}$. After cooling to room temperatures, the films were taken out and kept in desiccators to ensure good drying. The produced films had an average thickness of $\approx 0.0053 \pm 0.001 \mathrm{~cm}$. The blends of $\mathrm{PVA}_{x}$ $/ \mathrm{PEG}_{100-x}$ were prepared with different ratios where $x$ had the values of $100,93,86,79$, and $72 \mathrm{wt} \%$.

\section{2. $U V$-visible measurements}

A UV-visible spectrophotometer (V-550 JASCO Corp, Japan) was used for the measurements of the UV-vis absorption spectra in the range of wavelengths 190-900 nm for the prepared film samples.

\subsection{Reflection measurements}

A UV-vis-NIR spectrometer (UV-3101 PC, Shimadzu Europe, Germany), was used to detect the reflection spectra in the range of wavelengths 190-900 nm.

\subsection{FTIR measurements}

A FTIR spectrometer (FTIR - 460 plus, JASCO Corp., Japan) was used to measure the FTIR spectra in the wave number range $4000-400 \mathrm{~cm}^{-1}$.

\section{Results and discussion}

\subsection{FTIR analysis}

FTIR spectroscopy was used to figure out the probable interaction between the functional groups of PVA and PEG. In a range of wave numbers from $4000 \mathrm{~cm}^{-1}$ to $400 \mathrm{~cm}^{-1}$, FTIR absorption spectra were measured for the various contents of blends of $\mathrm{PVA} / \mathrm{PE}$ as shown in Fig. 1. For PVA, Fig. 1 and Table I illustrate a characteristic absorption band in the range of $3000-3400 \mathrm{~cm}^{-1}$ that appeared also in all the blends which is ascribed to the symmetrical stretching vibrations of $\mathrm{O}-\mathrm{H}$ groups. As listed in Table I, the bands existing at $2930 \mathrm{~cm}^{-1}$ and $1711 \mathrm{~cm}^{-1}$ are attributed to $-\mathrm{H}$ asymmetric stretching vibrations of the alkyl groups and to the $\mathrm{C}=\mathrm{O}$ stretching vibrations of the vinyl acetate groups of PVA, respectively. The $\mathrm{CH} 2$ bending group and $\mathrm{CH} 2$ wagging vibrations of PVA appeared at $1428 \mathrm{~cm}^{-1}$ and $1331 \mathrm{~cm}^{-1}$,

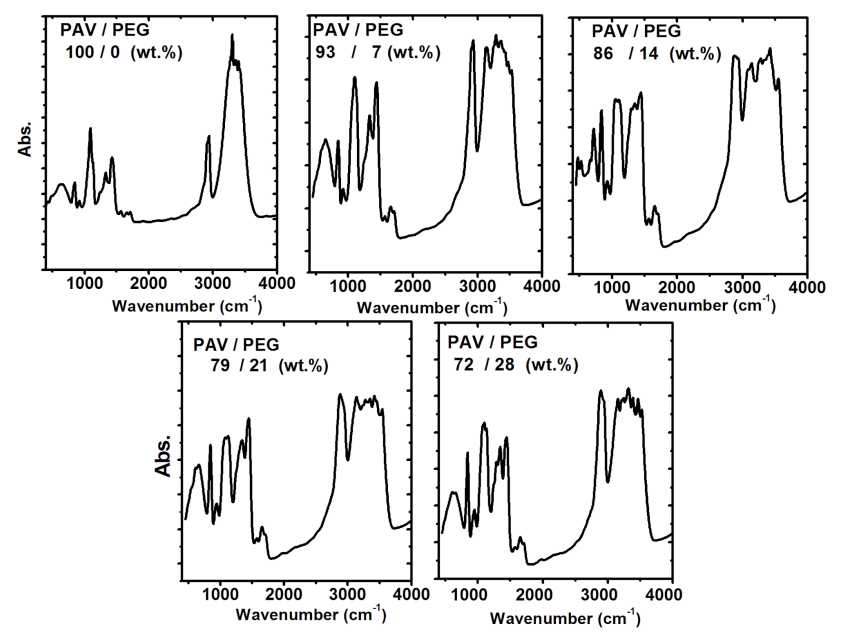

Fig. 1. FTIR spectra for pure PVA and various ratios of blends of PVA/PEG (wt\%).

respectively. Also, the observed band at $1093 \mathrm{~cm}^{-1}$ was ascribed to the $-\mathrm{O}$ stretching vibrations of acetyl groups of PVA.

In the case of PEG, Table I shows some of its characteristic bands. The $\mathrm{C}-\mathrm{OH}$ groups of PEG were observed at $942 \mathrm{~cm}^{-1}$ and $948 \mathrm{~cm}^{-1}$ only for its high contents as in the blends of (PVA/PEG 79/21 wt\%) and (PVA/PEG $72 / 28$ wt $\%)$, respectively. However, the skeletal vibration group of PVA, which was observed at the wave numbers 843 and $921 \mathrm{~cm}^{-1}$, disappeared after blending different contents of PEG with PVA. The stretching vibrations band of the $\mathrm{C}-\mathrm{C}$ group was detected nearly at $844 \mathrm{~cm}^{-1}$ after blending PVA with different contents of PEG while it was not observed in case of pure PVA. Also, the $\mathrm{C}-\mathrm{H}$ stretching vibrations band was observed in the range of 2933-2893 $\mathrm{cm}^{-1}$ depending on the quantity of PEG in the blends $[9,10]$. The observed shift in the positions of some functional groups of both PVA and PEG in the blends, as the bands of $\mathrm{CH} 2$ wagging vibrations, $\mathrm{C}=\mathrm{O}$ symmetric stretching, $\mathrm{CH}_{2}$ bending, $\mathrm{CH}_{2}$ wagging vibrations and $\mathrm{C}-\mathrm{H}$ stretching vibrations, indicates the interaction between their chains and the presence of polymeric structure changes.

\subsection{Optical absorption spectra}

The study of the optical absorption spectra is one of the most interesting methods for developing an understanding of the structure and energy gap of polymeric materials $[11,12]$. The measurements of the optical absorption were carried out to detect the effect of these ratios of embedding the PEG in the PVA. Figure 2a illustrates the absorption spectra for all of PVA/PEG blends in the UV-vis region 190-900 $\mathrm{nm}$. The measured spectra show an absorption band (shoulder) at about $276 \mathrm{~nm}$ for PVA and its position exhibited a very slightly blue shift with increasing PEG content in the blends becoming $280 \mathrm{~nm}$ for PVA/PEG 72/28 wt\%. These observed shoulders are attributable to the absorption by simple carbonyl groups along the PVA chain [13]. 
The wave numbers of assignments of the absorption bands deduced from FTIR spectra for different con-

TABLE I centrations of PVA/PEG (wt\%)

\begin{tabular}{c|c|c|c|c|c|c|c|c|c}
\hline \hline $\begin{array}{c}\mathrm{PVA} / \mathrm{PEG} \\
\text { [wt\%] }\end{array}$ & $\begin{array}{c}\mathrm{C}=\mathrm{O} \\
\text { symmetric } \\
\text { stretching }\end{array}$ & $\begin{array}{c}\mathrm{CH}_{2} \\
\text { bending }\end{array}$ & $\begin{array}{c}\mathrm{C}-\mathrm{O} \\
\text { stretching }\end{array}$ & $\begin{array}{c}\mathrm{C}-\mathrm{OH} \\
\text { bending }\end{array}$ & $\begin{array}{c}\mathrm{C}-\mathrm{H} \\
\text { stretching } \\
\text { vibrations }\end{array}$ & $\begin{array}{c}\mathrm{O}-\mathrm{H} \\
\text { symmetrical } \\
\text { stretching }\end{array}$ & $\begin{array}{c}\mathrm{C}-\mathrm{C} \\
\text { stretching } \\
\text { vibrations }\end{array}$ & $\begin{array}{c}\text { Skeletal } \\
\text { vibration }\end{array}$ & $\begin{array}{c}\mathrm{CH}_{2} \\
\text { wagging } \\
\text { vibrations }\end{array}$ \\
\hline $100 / 0$ & 1711 & 1428 & 1093 & - & 2930 & $3000-3400$ & - & $843-921$ & 1331 \\
$93 / 07$ & 1707 & 1438 & 1100 & - & 2933 & $3000-3400$ & 844 & - & 1331 \\
$86 / 14$ & 1705 & 1445 & 1105 & - & 2872 & $3000-3400$ & 841 & - & 1350 \\
$79 / 21$ & 1703 & 1445 & 1120 & 942 & 2881 & $3000-3400$ & 844 & - & 1338 \\
$72 / 28$ & 1707 & 1443 & 1098 & 948 & 2893 & $3000-3400$ & 844 & - & 1346
\end{tabular}
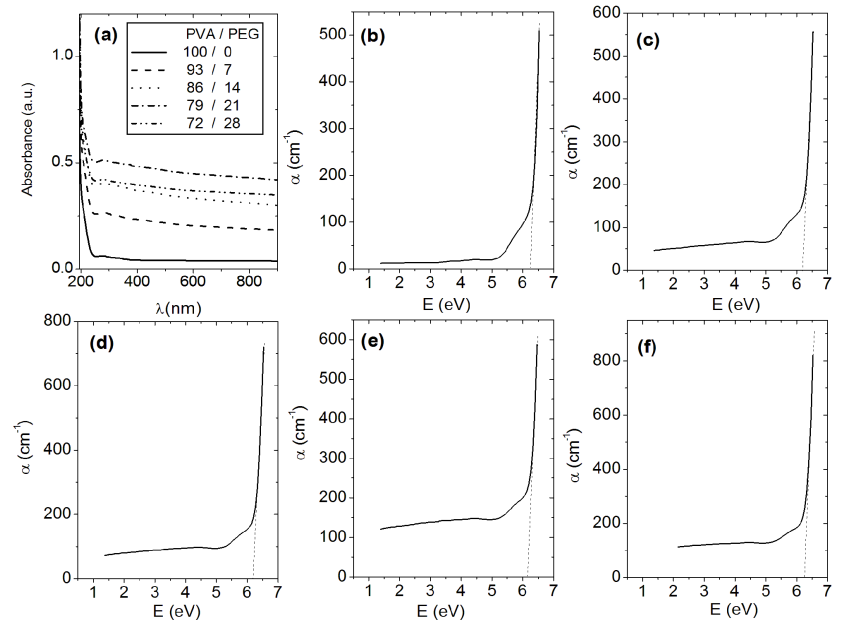

Fig. 2. (a) UV-visible absorption spectra for the various ratios of the PVA/PEG blends. The variation of the absorption coefficient $\alpha$ versus photon energy for (b) pure PVA, (c) PVA/PEG 93/07 wt\%, (d) PVA/PEG 86/14 wt\%, (e) PVA/PEG 79/21 wt\%, and (f) PVA/PEG $72 / 28 \mathrm{wt} \%$.

The variation of the absorption coefficient $\alpha\left(\mathrm{cm}^{-1}\right)$ with energy $E(\mathrm{eV})$ acts as a significant identification tool for polymeric materials providing optical information about them. The absorption coefficient $\alpha\left(\mathrm{cm}^{-1}\right)$ was estimated according to Eq. (1) [14]:

$$
\alpha(\nu)=2.303 \frac{\text { absorbance }}{\text { sample_thickness }} .
$$

Figure 2b-f illustrates the variation of $\alpha\left(\mathrm{cm}^{-1}\right)$ with $E(\mathrm{eV})$ for all the blends; they all showed an increase in the absorption near the edge which is a result of the creation of neutral excitations and/or the transition of electrons from the valence band to the conduction band. Figure $2 \mathrm{~b}-\mathrm{f}$ shows a steep rise in $\alpha$ near the absorption edge and there is a straight line relationship versus $E$ that can be determined in the high $\alpha$-region. The absorption edge values were determined from the intercept of an extrapolation to zero absorption with the photon energy axis [15]. As shown in Table II the calculated values of the absorption edges decreased with increasing PEG content up to the blend of (PVA/PEG $79 / 21$ wt $\%$ ) followed by an increase for the blend of (PVA/PEG 72/28 wt\%).
TABLE II

Absorption edge $A_{e d g}$, direct $E_{d g}$ and indirect $E_{i g}$ energy gap, the estimated energy gap $E_{g}$ from $\varepsilon_{\mathrm{i}}-E$ plot and the Urbach energy $E_{\mathrm{U}}$ estimated for various ratios of PVA/PEG (in wt\%)

\begin{tabular}{c|c|c|c|c|c}
\hline \hline $\begin{array}{c}\text { PVA/PEG } \\
{[\mathrm{wt} \%]}\end{array}$ & $\begin{array}{c}A_{e d g} \\
{[\mathrm{eV}]}\end{array}$ & $\begin{array}{c}E_{d g} \\
{[\mathrm{eV}]}\end{array}$ & $\begin{array}{c}E_{i g} \\
{[\mathrm{eV}]}\end{array}$ & $\begin{array}{c}E_{g} \\
{[\mathrm{eV}]}\end{array}$ & $\begin{array}{c}E_{\mathrm{U}} \\
{[\mathrm{eV}]}\end{array}$ \\
\hline $100 / 0$ & 6.247 & 6.351 & 5.267 & 5.242 & 0.228 \\
$93 / 7$ & 6.195 & 6.324 & 4.913 & 4.942 & 0.248 \\
$86 / 14$ & 6.182 & 6.304 & 4.818 & 4.822 & 0.257 \\
$79 / 21$ & 6.155 & 6.292 & 4.732 & 4.714 & 0.299 \\
$72 / 28$ & 6.256 & 6.341 & 4.801 & 4.800 & 0.272
\end{tabular}

\subsection{Skin depth}

Skin depth $\delta$ is also known as the penetration depth and it describes the distance that an electromagnetic wave can penetrate into a polymeric material. The skin depth was estimated as a function of wavelength by using the following relation [16]:

$$
\lambda \delta(\lambda)=\frac{1}{\alpha},
$$

where $\alpha$ is the absorption coefficient. Figure 3a shows the variation of the skin depth $\delta$ with wavelength for the various PVA/PEG blend ratios. For all the studied films the skin depth increased as the wavelength increased. However, by increasing the content of PEG in the PVA/PEG blend, the skin depth showed an obvious decrease in its values due to an increase in the absorption possibility. Consequently, we can conclude that skin depth was transmittance related [16].

\subsection{Interband transitions}

The Davis and Mott formula (Eq. (3)) describes the absorption coefficient $\alpha$ for a simple parabolic band as a function of photon energy in the case a radiation quantum is absorbed by a material; as used by one of us previously [17]:

$$
\alpha E=B\left(E-E_{g}\right)^{m},
$$

where $B$ is a constant that depends on the transition probability, $E_{g}$ is the optical band gap, $E$ is the photon energy and $m$ is an index with the values of $2,3,1 / 2$ or $3 / 2$, depending on the nature of the electronic transition responsible for the absorption. The dependence of $(a E)^{2}$ on the photon energy $E(\mathrm{eV})$ for the various PVA/PEG 

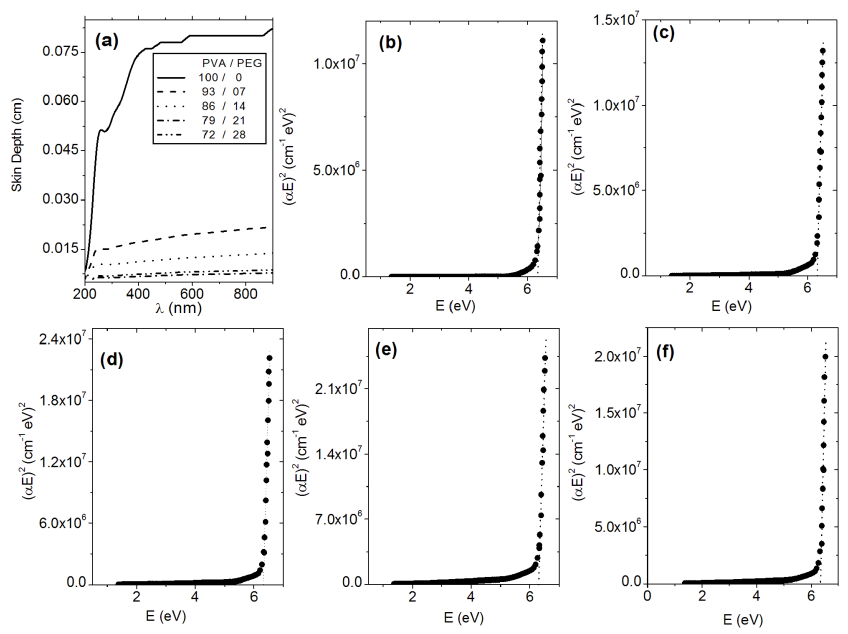

Fig. 3. (a) The skin depth versus the wavelength for the various ratios of PVA/PEG blends. The dependence of $(\alpha E)^{2}$ on the photon energy for (b) pure PVA, (c) PVA/PEG 93/07 wt\%, (d) PVA/PEG 86/14 wt\%, (e) PVA/PEG 79/21 wt\%, and (f) PVA/PEG 72/28 wt\%.

blends are shown in Fig. 3b-f all of which exhibit a linear behavior that proves the existence of the direct transition (i.e., for $m=\frac{1}{2}$ ). The optical gap was estimated from the intercept on the energy axis of the linear fit to the large energy data of the plots.

However, plotting $(a E)^{1 / 2}$ versus the photon energy $E(\mathrm{eV})$ as shown in Fig. 4, indirect transition (i.e., for $m=2$ ), the plots can be is resolved into two distinct straight-line portions. The straight line obtained at lower photon energies, corresponding to phonon-absorption process, cuts the energy axis at $E_{i g}-E_{p}$. The other line represents the dependence in the high energy range, corresponding to a phonon-emission process, and cuts the energy axis at $E_{i g}+E_{p}$. From the energy intercept of the two straight line portions, the value of $E_{i g}$ can be estimated [15]. Table II shows a decrease in the values of energy gaps of both the direct and indirect transitions, with increasing PEG content embedded in PVA up to the blend PVA/PEG 79/21 after which they increased. The decrease in the energy gap values is attributed to the formation of the localized states in the band gap by embedding PEG in PVA [18]. The formed localized states act as trapping and recombination centers which results in an alteration in the optical band gap. Also, the change in the PVA structure as a result of increasing the disorder degree by adding PEG can explain the decrease in the direct and indirect optical band gap [19].

In amorphous and disordered material, the observed Urbach tail plays an important role in describing well their properties of electronic transport. The strains in the network of the material, which are enough to force the states into the forbidden gap, result in the formation of the band tail states that decay exponentially in the band gap. Thus, it is noted that the Urbach tail acts as an indicator of the defect levels which exist in the

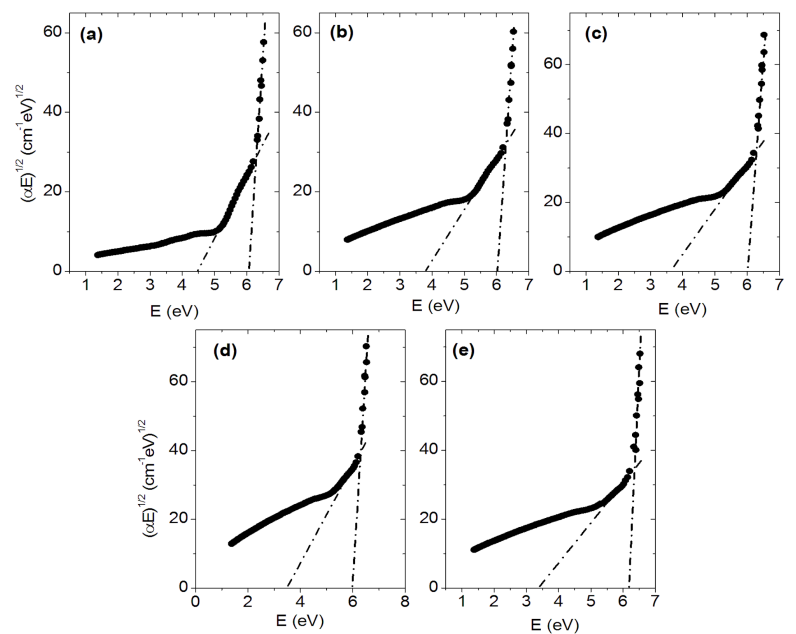

Fig. 4. The dependence of $(\alpha E)^{1 / 2}$ on the photon energy for (a) pure PVA, (b) PVA/PEG 93/07 wt\%, (c) PVA/PEG 86/14 wt\%, (d) PVA/PEG 79/21 wt\% and (e) PVA/PEG $72 / 28 \mathrm{wt} \%$.

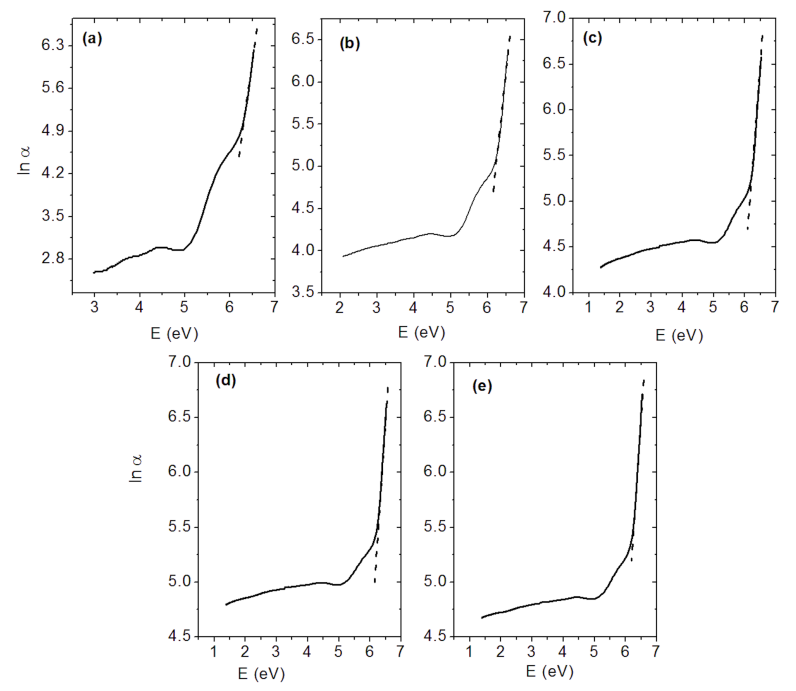

Fig. 5. The dependence of $\ln (\alpha)$ on the photon energy for (a) pure PVA, (b) PVA/PEG 93/07 wt\%, (c) PVA/PEG 86/14 wt\%, (d) PVA/PEG 79/21 wt\%, and (e) PVA/PEG $72 / 28 \mathrm{wt} \%$.

forbidden band gap. In this region the absorption process is ascribed to the transitions between extended states in one band and localized states in the exponential tail of the other band [19]. The Urbach energy was calculated from the following empirical formula that we developed previously [20]:

$$
\alpha=\alpha_{0} \exp \left(E / E_{\mathrm{U}}\right),
$$

where $\alpha_{0}$ is a constant and the Urbach energy $E_{\mathrm{U}}$ corresponds to the band tail width of the localized states in the band gap. The straight lines observed in plotting $\ln (\alpha)$ versus energy $E$ as shown in Fig. 5, indicate that the absorption spectra of the different blends of 
(PVA/PEG) confirm the rule of Urbach. Table II lists the calculated values for $E_{\mathrm{U}}$ for all of the samples which were estimated from the reciprocal of the slopes of the linear portions of the plots in Fig. 5. The increase in the $E_{\mathrm{U}}$ values with increasing the content of PEG in PVA/PEG, as illustrated in Table II, can be an indication of increase of the amorphous phase in the blends. However, in case of PVA, the lesser value of $E_{\mathrm{U}}$ in comparison with those of the blends means that the PVA had a wider energy band gap [21].

\subsection{Optical constants}

One of the fundamental properties of polymeric materials is their refractive index due to its important relationship with the local electric field and the electronic polarizability inside the material. Recently, great attention has been paid to enhancing the refractive index of a transparent polymer by blending it with another polymer in order to use it for production of photonic applications [22, 23]. The refractive indexes of our PVA/PEG blends were calculated from the reflection and absorption spectra, in a range of wavelengths $190-900 \mathrm{~nm}$, by using the following relationship [14]:

$$
n=\left(\frac{1+R}{1-R}\right)+\sqrt{\frac{4 R}{(1-R)^{2}}-k^{2}},
$$

where $R$ is the specular reflection and $k$ is the extinction coefficient corresponding to the imaginary part of the complex refractive index that can be calculated from Eq. (6) [14]:

$$
k=\frac{\alpha \lambda}{4 \pi}
$$

where $\alpha$ is the absorption coefficient and $\lambda$ is the wavelength. Figure 6 a shows typical dispersion curves of the refractive index of the various contents of PVA/PEG blends showing a slow decrease of the refractive index with increase of the wavelength. In Fig. 6a the PVA refractive index increases with increase of the content of PEG in the PVA/PEG blends up to PVA/PEG $79 / 21 \mathrm{wt} \%$ above which it decreased. The increase of the refractive index of the PVA/PEG blends compared to that of the pure PVA can be an indication of an increasing density of the blends which results in developing the interatomic spacing according to the famous formula of the Clausius-Mossotti [24, 25].

The loss in the electromagnetic energy, as a result of scattering and absorption per unit thickness, when the electromagnetic wave propagates through the material, is described by the extinction coefficient $k$. The obvious variation of the extinction coefficient $k$ with wavelength, especially at high wavelengths as shown in Fig. 6b, gives evidence of light photon-polymeric medium interaction [26].

The well known formula for the complex dielectric function is $\varepsilon=\varepsilon_{\mathrm{r}}+\mathrm{i} \varepsilon_{\mathrm{i}}$, where $\varepsilon_{\mathrm{r}}$ is the real part and $\varepsilon_{\mathrm{i}}$ is the imaginary part of dielectric constant. The real part of dielectric constant is associated with the dispersion which depends mainly on the motion of electrons in

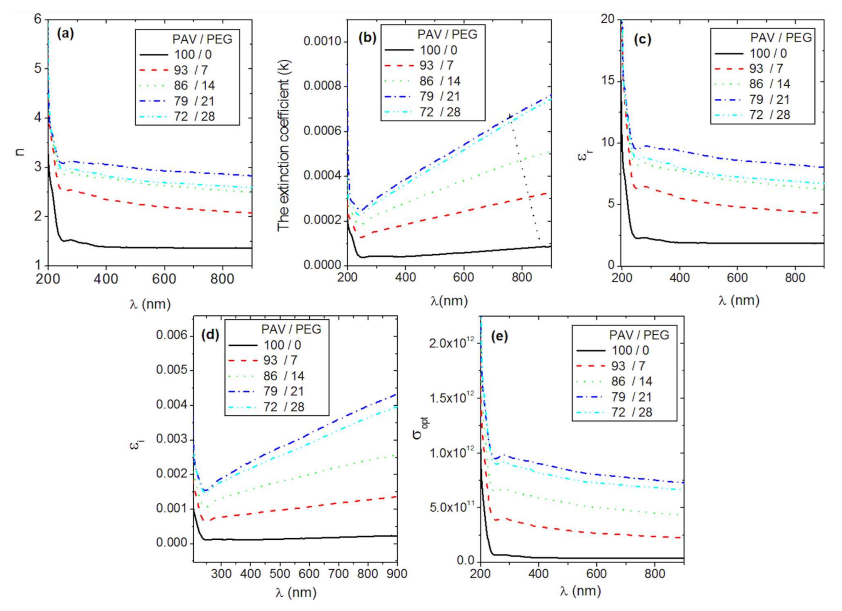

Fig. 6. (a) Variation of the refractive index with the wavelength for various ratios of the PVA/PEG blends. (b) The variation of the extinction coefficient $k$ with the wavelength for the various ratios of the PVA/PEG blends. The variation of the real part (c), the imaginary part of the dielectric constant (d), and the optical conductivity (e) with the wavelength for the various ratios of the PVA/PEG blends.

optical medium through the transmission of light. On the other hand, the imaginary part is responsible of the dissipative rate of electromagnetic wave propagation in medium. The real and imaginary parts of dielectric constant are described by the following equations in terms of the refractive index and extinction coefficient [24]:

$$
\begin{aligned}
& \varepsilon_{\mathrm{r}}=n^{2}-k^{2}, \\
& \varepsilon_{\mathrm{i}}=2 n k .
\end{aligned}
$$

Figure $6 \mathrm{c}$ and d shows the dependence of $\varepsilon_{\mathrm{r}}$ and $\varepsilon_{\mathrm{i}}$, respectively, on the photon energy $E(\mathrm{eV})$ for all the blends of PVA/PEG. The values of both $\varepsilon_{\mathrm{r}}$ and $\varepsilon_{\mathrm{i}}$ increased with increase of the PEG content in the PVA/PEG blends and then decreased for the highest content of the PEG embedded in PVA (PVA/PEG 72/28 wt\%). The calculated values of $\varepsilon_{\mathrm{r}}$ were higher than these of $\varepsilon_{\mathrm{i}}$, as $\varepsilon_{\mathrm{r}}$ is based on the values of $n^{2}$ while $\varepsilon_{\mathrm{i}}$ depends on both the $n$ and $k$ values with $k$ being affected by the absorption coefficient variations [27]. Based on the well-known Penn model exhibits the dependence of the real part of the dielectric constant $\varepsilon_{\mathrm{r}}$ on the optical band gap $E_{g}$ varies according to Eq. (9) [28]:

$$
\varepsilon_{\mathrm{r}}(0) \approx 1+\left(\frac{\hbar \omega_{p}}{E_{g}}\right)^{2}
$$

Equation (9) shows an inverse relationship between $\varepsilon_{\mathrm{r}}$ and $E_{g}$ which is in a good agreement with the results of this study since PVA had the lowest value of $\varepsilon_{\mathrm{r}}$ as shown in Fig. 6c while it had the highest value of $E_{g}$ (the direct $E_{d g}$ and indirect $E_{i g}$ ) as tabulated in Table II.

In addition, the interaction between the blends and the electromagnetic radiation was confirmed by the observed increase in the values of $\varepsilon_{\mathrm{i}}$ with increasing wavelength. 


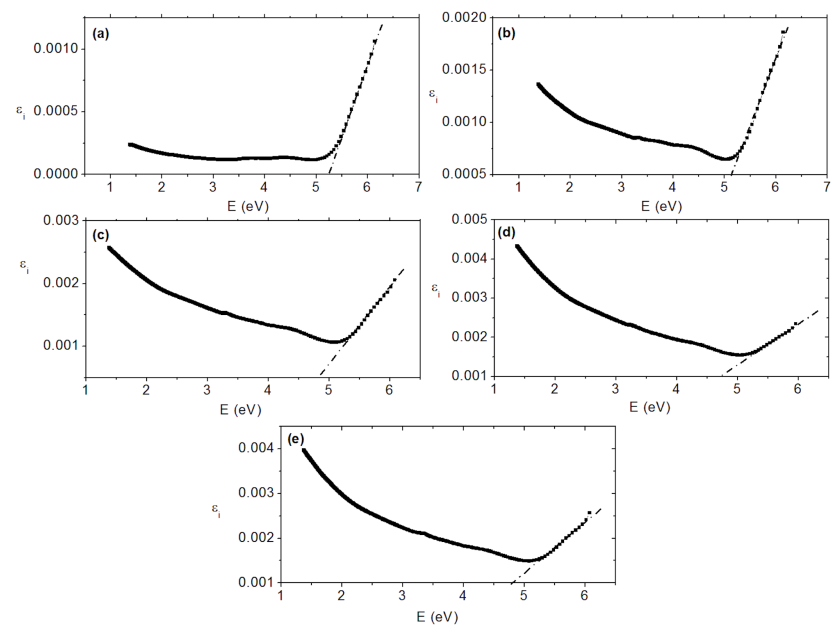

Fig. 7. The dependence of the imaginary part of the dielectric constant $\varepsilon_{\mathrm{i}}$ on the photon energy for (a) pure PVA, (b) PVA/PEG 93/07 wt\%, (c) PVA/PEG 86/14 wt\%, (d) PVA/PEG 79/21 wt\%, and (e) PVA/PEG $72 / 28 \mathrm{wt} \%$.

The interband absorption process describes the electron transition process between the bands of the solid materials. However, the absorption edge originates from the onset of optical transitions across the fundamental band gap of a solid [19].

Basically, $\varepsilon_{\mathrm{i}}$ distinguishes the transfer of electrons from occupied to unoccupied states [19]. It was found that there was a strong relationship between the imaginary part of the dielectric constant and the absorption coefficient. A short time ago it was found that the fundamental absorption edge determined from the dielectric loss versus photon energy was so close to that obtained from the Tauc formula which was proved by $\mathrm{Yu}$ et al. [29]. For all of our different blends the optical dielectric loss versus photon energy is shown in Fig. 7; all samples illustrated a near linear behavior at high energy. The close similarity between the optical band gap values determined from Fig. 4 and the values estimated from Fig. 7, as shown in Table II, indicates that an indirect allowed transition was dominant in all the samples. Also, estimating the optical band gap values was successfully achieved by two powerful ways, the Tauc formula and the gained fundamental absorption edge from the dielectric loss vs. photon energy, as presented in this study.

The optical conductivity $\sigma_{o p}$, describes the optical response of the tested sample material and according to the Gaussian system of units, it has the dimensions of frequency $\sigma_{o p}$ was calculated from the following formula [30]:

$$
\sigma_{o p}=\frac{\alpha n c}{4 \pi},
$$

where $c$ is the velocity of light in space, $\alpha$ is the absorption coefficient and $n$ is the refractive index. The variations of the optical conductivity $\sigma_{o p}$ with the incident energy of the photons for the various blends of
PVA/PEG are shown in Fig. 6e. A considerable increase in the values of $\sigma_{o p}$ was observed with increasing PEG content in the blend up to PVA/PEG $79 / 21 \mathrm{wt} \%$, then it decreased. The increase in the values of $\sigma_{o p}$ is a result of increasing the density distance by increasing the content of PEG. On the other hand, the observed decrease of $\sigma_{o p}$ of PVA/PEG $72 / 28 \mathrm{wt} \%$ is due to the decreased density of localized states in the gap as a consequence of the decrease in the density of defect states [31].

\section{Conclusions}

A simple casting method was used to prepare different ratios of PVA/PEG blends. The existence of an interaction between PVA and PEG was demonstrated for the blends by the shift in the absorption spectra to lower photon energy. The decrease in the direct and indirect energy gaps of PVA by blending it with PEG is suggested to be a result of creation of subband gaps between the valence and conduction bands. The increase in the values of the Urbach energy of PVA by blending with PEG is a result of forming disordered blends. Increasing the density of PVA by adding PEG explains the enhancement in the refractive index of the blends than PVA. For all the blends, the increase of the extinction coefficient at high wavelength is ascribed to scattering and losing small photon energy. The complex optical dielectric functions were effectively used to confirm that the electron transition that was responsible for the absorption process was an indirect allowed transition. The optical band gap and the real and imaginary parts of the optical dielectric constants were all increasing while the skin depth was decreasing with increase of the PEG contents. The shift in the position of the functional group of PVA and PEG gives an indication of the possible interaction between them.

\section{References}

[1] I. Plesa, P.V. Notingher, S. Schlögl, C. Sumereder, M. Muhr, Polymers 8, 173 (2016).

[2] Z.H. Esfahani, M. Ghanipour, D. Dorranian, J. Theor. Appl. Phys. 8, 117 (2014).

[3] D.J. Walsh, S. Rostami, Adv. Polym. Sci. 70, 119 (1985).

[4] K. Ramamohan, V.B.S. Achari, A.K. Sharma, Lu Xiuyang, Ionics 21, 1333 (2015).

[5] M.L. Hallensleben Polyvinyl compounds, others, in: Ullmann's Encyclopedia of Industrial Chemistry, Wiley-VCH, Weinheim 2000.

[6] S. Zahedi, D. Dorranian, Opt. Rev. 20, 36 (2013).

[7] A.M. El Sayed, H.M. Diab, R. El-Mallawany, J. Polym. Res. 20, 255 (2013).

[8] N. Tasić, Z. Branković, Z. Marinković-Stanojević, G. Branković, Sci. Sinter. 44, 365 (2012).

[9] K. Deshmukh, M.B. Ahamed, K.K. Sadasivuni, D. Ponnamma, R.R. Deshmukh, S.K.K. Pasha, M.A. AlMaadeed, J. Polym. Res. 23, 159 (2016). 
[10] R.M. Ahmed, Int. J. Mod. Phys. B 28, 1450036 (2014).

[11] A.F. Mansour, R.M. Ahmed, A.H. Bassyouni, G.M. Nasr, Int. J. Polym. Mater. 56, 207 (2007).

[12] G. Ross, A.W. Birley, J. Phys. D Appl. Phys. 6, 795 (1973).

[13] A. El-Khodary, J. Phys. B At. Mol. Phys. 404, 1287 (2009).

[14] R.M. Ahmed, Int. J. Photoenergy 2009, 150389 (2009).

[15] R.M. Ahmed, S.M. El-Bashir, Int. J. Photoenergy 2011, 801409 (2011).

[16] W.A. Al-Taa'y, S.F. Oboudi, E. Yousif, M. Abdul Nabi, R.M. Yusop, D. Derawi, Adv. Mater. Sci. Eng. 2015, 913260 (2015).

[17] R.M. Ahmed, Int. J. Polym. Mater. 57, 969 (2008).

[18] R.M. Ahmed, Polym. Compos. 38, 749 (2017).

[19] S.B. Aziz, H.M. Ahmed, A.M. Hussein, A.B. Fathulla, R.M. Wsw, R.T. Hussein, J. Mater. Sci. Mater. Electron. 26, 8022 (2015).

[20] A.F. Mansour, R.M. Ahmed, A.H. Bassyouni, G. Nasr, Int. J. Polym. Mater. 56651 (2007).

[21] S. Prasher, M. Kumar, S. Singh, Int. J. Polym. Anal. Charact. 19, 204 (2014).
[22] A.M. Shehap, D.S. Akil, Int. J. Nanoelectron. Mater. 9, 17 (2016).

[23] N. Tanio, J. Adhes. Soc. Jpn. 47, 203 (2011) (in Japanese).

[24] M. Ghanipour, D. Dorranian, J. Nanomater. 2013 , 897043 (2013).

[25] C. Kittle, Introduction to Solid State Physics, Vol. 405, Wiley, New York 1971.

[26] S.B. Aziz, O.G. Abdullah, A.M. Hussein, R.T. Abdulwahid, M.A. Rasheed, H.M. Ahmed, S.W. Abdalqadir, A.R. Mohammed, J. Mater. Sci. Mater. Electron. 28, 7473 (2017).

[27] T.J. Alwan, Malays. Polym. J. 5, 204 (2010).

[28] S.B. Aziz, J. Nonmater. 7, 444 (2017).

[29] L. Yu, D. Li, S. Zhao, G. Li, K. Yang, Materials 5 , 2486 (2012)

[30] B.H. Rubee, S. Abd AlHussien, Int. J. Sci. Res. 5, 1794 (2016)

[31] D. Singh, S. Kumar, R. Thangaraj, J. Phase Transit. 87, 206 (2014) 